\title{
What is the origin of the very high energy (VHE) emission from AP Librae?
}

\section{Agniva Roychowdhury, ${ }^{a, *}$ Eileen T. Meyer, ${ }^{a}$ Markos Georganopoulos ${ }^{a, b}$ and Peter Breiding $^{c}$}

${ }^{a}$ Department of Physics, University of Maryland Baltimore County, 1000 Hilltop Circle, Baltimore, MD 21250, USA

${ }^{b}$ NASA Goddard Space Flight Center, Code 663, Greenbelt, MD 20771, USA

${ }^{c}$ Department of Physics and Astronomy, West Virginia University, P.O. Box 6315, Morgantown, WV 26506, USA

E-mail: agniva.physics@umbc.edu

The high-energy spectral component in blazars is usually attributed to various inverse Compton scattering processes in the relativistic jet, but has not been clearly identified in most cases due to degeneracies in physical models. AP Librae, a low-synchrotron-peaking BL Lac object (LBL) detected in 2015 by H.E.S.S. at TeV energies, has an extremely broad high-energy spectrum, covering $\sim 9$ decades in energy. Standard synchrotron self-Compton models generally fail to reproduce the VHE emission, which has lead to the suggestion that it might arise not from the blazar core, but on kilo-parsec scales from inverse-Compton scattering of cosmic microwave background (CMB) photons by a still-relativistic jet. Such a model makes specific predictions for the level of infrared emission from the kpc-scale jet which we can now rule out with newly obtained Hubble imaging. Sub-mm ALMA imaging has also revealed the presence of a $\sim 500$ pc quasi-thermal circumnuclear disk, which is the first detection of a torus in a BL Lac. We find that the VHE emission can be reproduced through inverse-Compton scattering of torus photons by electrons in the sub-kpc jet. This explanation could be further evaluated with deep and high dynamic range imaging in the sub-mm and far infrared and continued monitoring of the source at $\mathrm{TeV}$ energies to test for variability.

\footnotetext{
*** 37th International Cosmic Ray Conference (ICRC2021), ***

*** 12-23 July $2021 * * *$

*** Berlin, Germany - Online ***
}

\footnotetext{
${ }^{*}$ Presenter
} 


\section{Introduction}

Blazars are radio-loud active galactic nuclei (AGN) with a relativistic jet aligned within a few degrees to our line of sight $[2,33]$. This causes Doppler boosting of the emission from the base of the jet (or 'core') creating a 'jet-dominated' blazar spectral energy distribution (SED) from radio to gamma-rays, which in $\log v \mathrm{~F}_{v}-\log v$ exhibits two broad components of nearly equal amplitude, the first peaking anywhere between $10^{12}-10^{17} \mathrm{~Hz}$ and the second at $\mathrm{GeV}$ to $\mathrm{TeV}$ energies $[6,18,34]$.

Although the cause of the lower-energy emission component in blazars is very well established as synchrotron radiation from relativistic electrons [e.g. 32], there is less certainty regarding the location and origin of the high-energy (X-ray to gamma-ray) emission from blazars. In most cases the same relativistic electrons responsible for synchrotron emission inverse Compton scatter either their own synchrotron photons (synchrotron self-Compton, SSC) or photons from an external field (external Compton or EC) to produce the GeV-TeV component of the SED [see e.g., 3]. Hadronic models are an alternative to the purely leptonic scenario where protons are accelerated to very high energies $(\gtrsim \mathrm{PeV})$ and can produce $\gamma$-rays via synchrotron radiation $[1,21,25]$ or indirectly via synchrotron plus Compton processes of secondary leptons produced in lepto-hadronic interactions $[1,15,16,22,26]$.

The blazar population can be divided phenomenologically into low-synchrotron-peaking (LSP, $v_{\text {peak }}<10^{14} \mathrm{~Hz}$ ), intermediate-synchrotron-peaking (ISP, $10^{14}<v_{\text {peak }}<10^{15} \mathrm{~Hz}$ ) and highsynchrotron-peaking HSP sources (HSP, $v_{\text {peak }}>10^{15} \mathrm{~Hz}$ ). Separately from the SED type, blazars with quasar-like emission lines (nearly all LSP sources) are known as flat-spectrum radio quasars (FSRQs) and those without broad lines (or any lines) are known as BL Lacs. AP Librae is a well-known nearby LSP BL Lac (LBL) at $z=0.049$ [3.8 kpc/4", 13] and one of the four LBLs detected at $\sim \mathrm{TeV}$ energies [10]. The X-ray to $\mathrm{TeV}$ component is unusually broad, extending over $\sim 9$ orders of magnitude in frequency. AP Librae also exhibits a resolved radio jet which has been detected in the X-rays with Chandra [4, 14], making it the only LBL source out of approximately 200 AGN known to host an X-ray emitting large-scale jet.

The broadness of the high-energy component in AP Librae relative to the synchrotron peak makes it difficult to model using standard single-zone SSC or EC models. Several efforts have been made to move beyond a simple SSC model and explain the TeV emission. Both [35] and [30] suggested that inverse Compton scattering off cosmic microwave background photons in the extended jet (the IC/CMB mechanism) could explain the high-energy LAT and H.E.S.S. emission, although the synchrotron emission of the large-scale jet was severely under-constrained in these works. Alternatively, a lepto-hadronic scenario with the $\mathrm{TeV}$ emission primarily arising from photo-hadronic processes in the core has been proposed [27].

For AP Librae, the potential physical scenarios and emission mechanisms laid out in previous works imply very different jet characteristics from jet composition and bulk velocity to the total energy content. Our aim in this study is to explain the $\mathrm{TeV}$ emission in AP Librae and in particular to test the clear predictions of the single-zone IC/CMB model using the Very Large Array (VLA), Atacama Large Millimeter/sub-millimeter Array (ALMA), the Hubble Space Telescope (HST), and the Fermi/LAT observatory. 

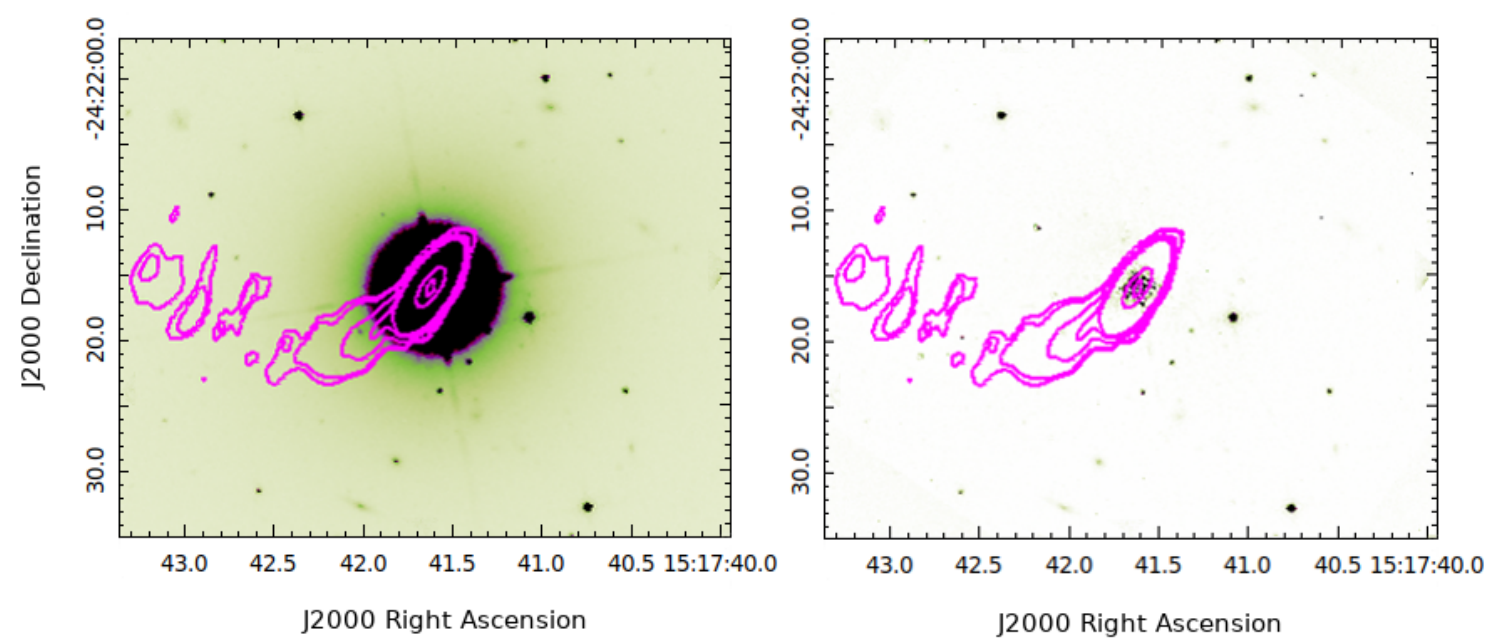

Figure 1: Drizzled HST/WFC3 images of AP Librae before (left) and after (right) GALFIT model subtraction, overlaid with VLA L-band contours. The subtracted image on the right shows no evidence of jet-related flux.

\section{Observations}

\subsection{Very Large Array}

We reduced several historical VLA observations of AP Librae, from 1.5 to $8.4 \mathrm{GHz}$, using the Common Astronomy Software Applications [CASA; 17]. We applied standard initial calibration to all the datasets. AP Librae is core-dominated and very bright in all radio imaging Previous radio imaging shows AP Librae to host a $\sim 20^{\prime \prime}$ long radio jet and our imaging deconvolution showed components in the large-scale jet within $1^{\prime \prime}$ from the peak core position. Further details have been noted in [29].

\subsection{Atacama Large Millimeter/sub-millimeter Array}

We analyzed a number of archival ALMA observations taken in bands 3-9 from 100 to 700 $\mathrm{GHz}$ to better constrain the synchrotron spectrum of the kpc-scale jet in AP Librae. The residual images after core-subtraction show both emission from the jet, as well as residual flux elongated in the NE-SW direction of size $\simeq 1 "(\sim 0.9 \mathrm{kpc})$ around the core, which is increasingly dominant with increase in frequency. We deduced from spectral modelling in Section 3.2 that this residual emission is quasi-thermal and is essentially a circumnuclear disk (CND)/large-scale torus. Further details will be explored in [29].

\subsection{Hubble Space Telescope}

AP Librae was observed with the Wide-field Camera 3 (WFC3) UVIS/IR imager on HST. Observations totaling 5016 seconds were taken on 15 February 2018 with the F160W filter which peaks at 1.5 microns.

In order to more clearly observe any possible jet-related emission in our near-IR imaging, it was necessary to model and subtract the host galaxy emission, the emission from the torus and the central point-source due to the bright blazar core. 
We used the publicly available GALFIT [GALaxy-FITting; 24] routine to model the galaxy (Sérsic), the torus (exponential disk) and the central point source (point spread function). A comparison of the resulting image using non-subtracted and subtracted frames is shown in Figure 1, with L-band VLA radio contours overlaid.

There is no indication of any jet-related flux in the HST imaging after subtraction. We confirmed this and derived an upper limit for the IR flux. Due to imperfections in the galaxy subtraction, we carefully sampled areas of the subtracted image to derive our $5 \sigma$ upper limit of $0.70 \mu \mathrm{Jy}$. Further, using the value of the magnitude photometric zeropoint for HST/WFC3 as 28.2 (see HST/WFC3 handbook) in Equation 5 in [24], we obtained the total integrated flux density of the exponential disk component, which we assume to be IR dust emission, as $1.27 \pm 0.10 \mathrm{mJy}$. The radius of this disk is $\simeq 6$ pixels, and with a plate scale of $\sim 0.12 \% /$ pixel for WFC3, it turns out to be $\simeq 0.7 \mathrm{kpc}$, consistent with ALMA observations. Further details can be found in [29].

\subsection{Fermi}

AP Librae has been detected by the Fermi/LAT and is listed in the 4FGL-DR2 point source catalog as 4FGL J1517.7-2422 with a detection significance of 95. We have analyzed over 12 years of continuous Fermi/LAT observations to derive an average spectrum for this source, and have also derived the minimum flux state using the progressive-binning analysis method first presented in [19]. Fermi/LAT event and spacecraft data were extracted using a $7^{\circ}$ region of interest (ROI), an energy cut of $50 \mathrm{MeV}-300 \mathrm{GeV}$, and a zenith angle cut of $90^{\circ}$. The time cuts included all available Fermi data at the time of analysis, with corresponding mission elapse time (MET) ranges of 239557417 to 626971271 (corresponding from 4 August 2008 to November 13 2020).

\section{Results and Discussion}

Our objective in this paper is to make indicative fits to the spectral energy distribution (SED) of AP Librae discussing the different possibilities of varying seed photon fields as a source of $\mathrm{TeV}$ emission. We first evaluate the IC/CMB model and thereafter discuss other photon fields as required.

\subsection{Evaluating the IC/CMB Model}

We present an updated spectral energy distribution for AP Librae in Figure 2, where all observations of the kpc-scale jet below X-ray energies as well as the Fermi/LAT observations are newly presented in this paper. The historical total source fluxes (dominated by the blazar core) shown in gray are taken from [35, hereafter Z16] and/or NED. We have used red data points for the kpc-scale jet fluxes and IR upper limit and orange triangles for the jet+dust fluxes measured by ALMA for the frequencies above $100 \mathrm{GHz}$ which are clearly dominated by the thermal component. An orange box has been used for the IR dust flux. The X-ray spectrum for the kpc-scale jet is also shown as red points [from 14]. In the GeV-TeV band we show the Fermi/LAT 12 yr average flux in dark gray and the minimum LAT fluxes in dark blue. Finally the H.E.S.S. TeV flux points are shown in magenta [from 10]. In this section, we will primarily focus on the CMB seed photon field and the validity of the IC/CMB model for the TeV emission. In following sections, we discuss the dust seed photon field in detail and its contribution to the broadband SED. 


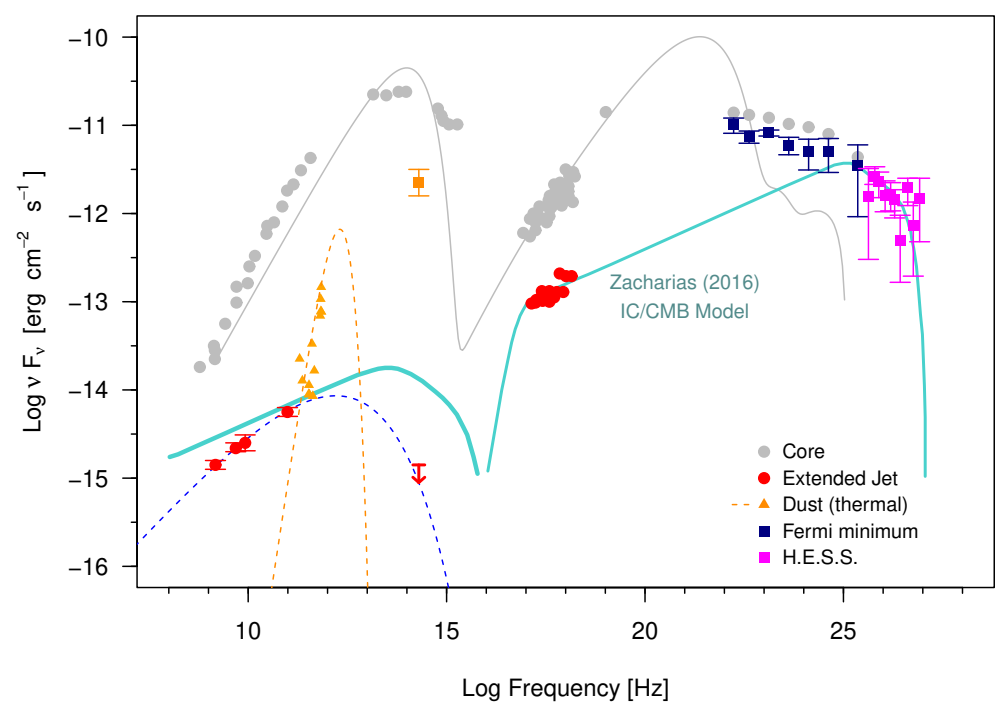

Figure 2: The spectral energy distribution of AP Librae. The bright blazar core dominates over the kpcscale jet from radio to X-rays and the data shown in gray are historical total source (i.e., core) fluxes. The corresponding gray solid line is a one-zone SED model for the core (see section 3.4). From our highresolution VLA, ALMA, and HST imaging campaign we have been able to more accurately sample the synchrotron spectrum of the X-ray emitting jet (red data points and upper limit with phenomenological power-law+exponential cutoff model as a blue dashed curve). The infrared upper limit (in red) in particular rules out the IC/CMB model of [35], shown as cyan curves. In addition, a fit to the orange ALMA data points (orange-dashed) has been indicated, which is a blackbody at temperature $25 \mathrm{~K}$, having typical size $100 \mathrm{pc}$. The corresponding dust flux in the IR is given as an orange box.

While we are able to separately resolve the kpc-scale jet at lower frequencies, in the GeV-TeV regime the observations shown in Figure 2 are for the entire source and it is not clear which zone of emission dominates. The light gray and cyan model curves are taken from Z16. The thick cyan curve is their synchrotron model for the large-scale jet and the thin cyan curve the corresponding IC/CMB emission proposed to explain the kpc-scale $\mathrm{X}$-ray and (total) $\mathrm{TeV}$ emission. The required synchrotron spectrum under this model is now clearly very discrepant considering the new data points. Since the inverse Compton spectrum for an external photon field is a copy of the synchrotron in frequency and luminosity [8], this simple one-zone IC/CMB model for the $\mathrm{TeV}$ emission can be ruled out [8]. However, more complex external Compton (EC) models, which may include IC/CMB, remain viable as we will show below in the following sections.In the following sections, we test different seed photon fields using spectral energy distribution (SED) modeling.

\subsection{Properties of the dust spectrum}

Based on the core-subtracted ALMA residual images, the dust emission appears to arise from a circumnuclear disk, possibly viewed at a high inclination. This is the first detection of large-scale dust emission in a BL Lac and is in marked contrast with studies that have otherwise failed to detect any dust emission from a torus in a large sample of BL Lacs [28]. However, detailed modelling of its structure [e.g., 7, 23, 31] is out of the scope of this paper and hence we will employ a very simple cylindrical model (with thickness and height) to model the emission spectrum. Observationally, this torus extends through few hundred parsecs and is a reservoir of seed photons for inverse Compton 
scattering in the sub-pc core as well as the extended kpc-scale jet. Hence it is very relevant in our pursuit of the origin of the VHE emission.

Using ALMA imaging [29] the approximate radius $R$ and thickness of the disk $H$ are 0.65 and $0.20 \mathrm{kpc}$ respectively, or $H \simeq 2 R / 5$. The inner radius $r_{i n}$ of the disk must connect with the pc-scale torus (if present) and for the purpose of modelling which we neglect this as $r_{i n}^{2} \ll R^{2}$. We find that the ALMA fluxes match a blackbody with the total luminosity of the disk $\mathrm{L}=(2 \pi R(R+H)) \sigma T^{4}$ with $T=25 \mathrm{~K}$ and $R=100 \mathrm{pc}$.

From HST imaging, we only have one observation in the IR, which equals a flux value $F_{\text {ir }} \simeq 1.27 \pm 0.10 \mathrm{mJy}$ at $10^{14.30} \mathrm{~Hz}$, which we convert into a luminosity using a Heaviside spectral flux density centred at $10^{14.30} \mathrm{~Hz}$ with width equal to that of the HST/WFC3 filter.

\subsection{Evaluating the IC-dust model}

The discussion in the previous section disproved a simple one-zone IC-CMB model for the origin of the $\mathrm{TeV}$ emission. In this section, we explore the possibility of electrons in the sub-kpc to kpc-scale jet IC-scattering dust photons to produce the $\gtrsim 100 \mathrm{GeV}$ emission.

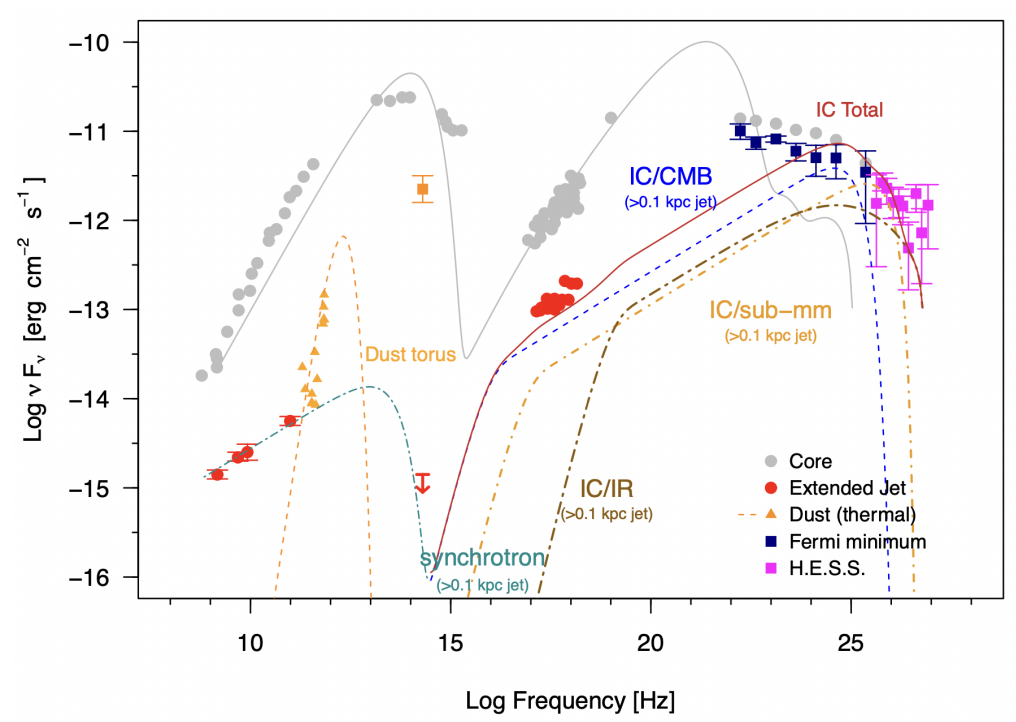

Figure 3: Data points are as in Figure 2. Orange fitted line is the blackbody fit to the ALMA fluxes for $\mathrm{T}=25 \mathrm{~K}$ and size $100 \mathrm{pc}$. Three different models of inverse-Compton scattering are shown: IC/CMB in blue dashed line, IC/IR in brown dot-dashed line and IC/ALMA (sub-mm) in orange dot-dashed line, while the extended jet synchrotron is plotted as a cyan dashed line. The gray line is the total SED of the core, including multi-order synchrotron self-Compton and IC/dust (IR and ALMA). It is clear that the total IC spectrum (red solid line) describes the $\mathrm{GeV}$ and most part of the $\mathrm{TeV}$ emission.

We use a simple one-zone homogeneous leptonic model, that includes Klein-Nishina effects, to understand the spectral energy distribution (SED) of AP Librae. We have three seed photon fields, namely, the CMB, the sub-mm and the IR dust. We find that the CMB photons are inverseCompton scattered to produce the low-energy end of the VHE spectrum while both the sub-mm and the infrared dust photons are IC-scattered to produce the TeV emission. The final SED is shown in Figure 3. The orange data points (error bars omitted to prevent clutter) describe the ALMA and the HST dust fluxes and the orange dashed line represent the $25 \mathrm{~K}$ blackbody fit with $100 \mathrm{pc}$ size, as 
discussed earlier. The dark dashed cyan curve is the synchrotron component from our SED model. All the high-energy components due to inverse Compton scattering have been marked, IC/CMB in blue, IC/ALMA(sub-mm) in orange and IC/IR in brown. The gray line is the spectrum of the blazar core. It is clear that the $\mathrm{GeV}$ emission can be represented by an IC/CMB model while the $\mathrm{TeV}$ is majorly produced by IC/sub-mm and IC/IR within the kpc-scale jet. It is also evident that this simple IC/CMB model underproduces the observed X-ray emission from the kpc-scale jet and although with more fine-tuning of our model parameters the discrepancy can be reduced, we omit it since the major aim of this work is to explain the $>100 \mathrm{GeV}$ emission. In fact, the origin of X-rays from large-scale jets is actually an open question and they may as well not be produced by IC/CMB in a single emitting region [e.g., 12, 20].

We illustrate pictorially in Figure 4 the origin and location of the VHE emission in AP Librae, based on our one-zone model. The mean de-projected location of the VHE emission is $0.7 \mathrm{kpc}$ along the jet which is $\sim$ few times the thickness $0.1 \mathrm{kpc}$ of the dusty disk/torus, implying the disk will not be $\gamma-\gamma$ opaque to the VHE emission. If this dusty disk extends down to the sub-pc scales, it will be opaque to a considerable fraction of the VHE emission originating from the pc-scale core ( $\tau_{\gamma \gamma} \gg 1$ using e.g., [9]) and this would simply rule out any pc-scale model of VHE emission from AP Librae. This can be further confirmed with deeper ALMA/JWST observations of the torus.

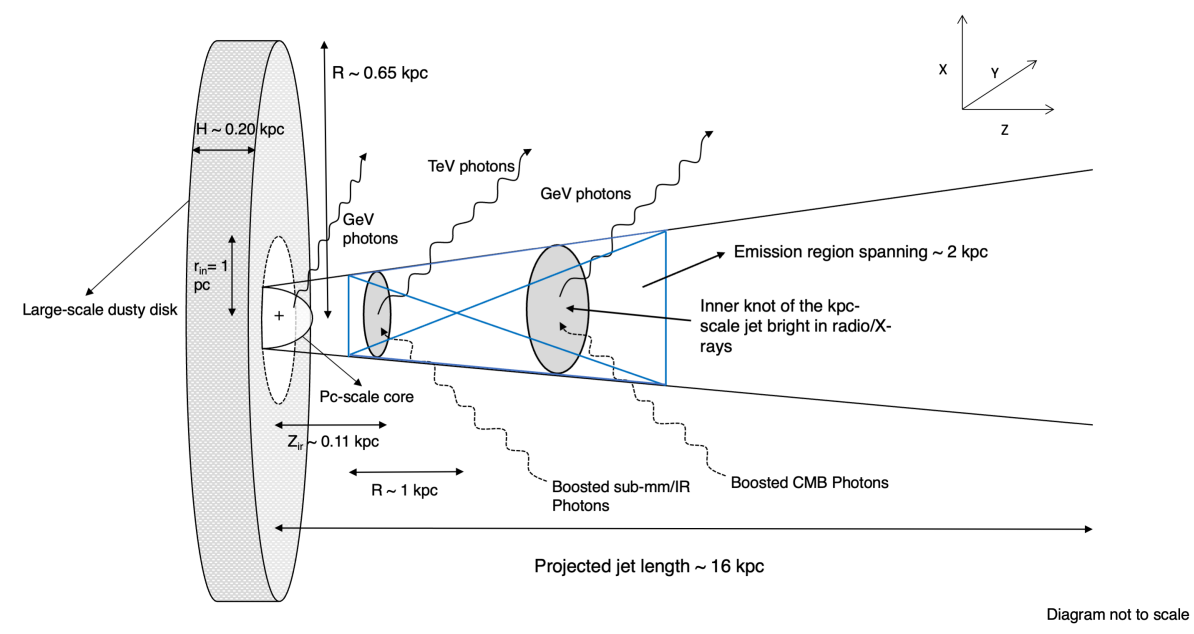

Figure 4: An illustration of the assumed morphology, emission mechanisms and locations as determined from observations and SED modelling. Figure shows the basic model of the dust disk surrounding a pc-scale core (with the jet apex marked with a cross), with the disk having approximate minimum radius as $r_{i n}$. The kpc-scale jet is shown to the right with the emission region size obtained from the SED model. The X-ray emission is from the kpc-scale jet [14], the $\mathrm{TeV}$ emission is produced at $\sim 0.11 \mathrm{kpc}$ along the kpc-scale jet by IC/Dust scattering, while the $\mathrm{GeV}$ emission is partly produced from the pc-scale core and mainly from the extended jet through IC/CMB scattering.

\section{Conclusion}

In this work, we have found evidence for a large-scale dusty torus in a BL Lac for the first time. This is in sharp contrast to previous observations, where e.g., [28] found no evidence of torus 
emission in a sample of $\sim 100$ WISE detected BL Lacs. On the contrary, FSRQs are the only blazars with existing tori emission [5]. Absence of a torus may be linked to weak accretion disk emission in BL Lacs [e.g., 11] or highly Doppler boosted jet emission that may obscure the detection of a dusty torus. In contrast, in AP Librae, if the kpc-scale torus extends all the way to pc-scales, this poses a new question for the theory of radio-loud AGN unification of BL Lacs and FSRQs. However, our observations are not deep enough and further clarity on the dust emission and thereafter the source of $\mathrm{TeV}$ emission can be obtained with new, deep spectral line and continuum IR and sub-mm observations. The IC/CMB and multiple hadronic models [27] can be tested through monitoring the source for $\mathrm{TeV}$ variability. Because of the scale of the resolved jet and the total steadiness of the CMB, the TeV emission resulting from IC/CMB in the kpc-scale jet should be absolutely non-variable. On the other hand, it is unclear to what extent the dust emission might vary and only through combined multi-wavelength imaging and spectroscopic campaigns can such mysteries be unravelled.

\section{Acknowledgments}

This work was supported by the HST-GO grant 15175.

\section{References}

[1] Aharonian. 2000, NewA, 5,377

[2] Blandford et al. 2019, ARA\&A, 57, 467

[3] Böttcher. 2007, ApSS, 309,95

[4] Cassaro et al. 1999, A\&AS, 139, 601

[5] Castignani, \& De Zotti. 2015, A\&A, 573, A125

[6] Fossati et al. 1998, MNRAS, 299, 433

[7] Fritzet al. 2006, MNRAS, 366, 767

[8] Georganopoulos et al. 2006, ApJL, 653, L5

[9] Ghisellini, \& Madau. 1996, MNRAS, 280, 67

[10] H. E. S. S. Collaboration et al. 2015, A\&A, 573, A31

[11] Hardcastle, \& Croston. 2020, NewAR, 88, 101539

[12] Harris, \& Krawczynski. 2006, ARA\&A, 44, 463
[13] Jones et al. 2009, MNRAS, 399, 683

[14] Kaufmann et al. 2013, ApJ, 776, 68

[15] Mannheim. 1993, PhRD, 48, 2408

[16] Mannheim et al. 1991, A\&A, 251, 723

[17] McMullin et al. 2007, in Astronomical Society of the Pacific Conference Series, Vol. 376, Astronomical Data Analysis Software and Systems XVI, ed. R. A. Shaw, F. Hill, \& D. J. Bell, 127

[18] Meyer et al. 2011, ApJ, 740, 98

[19] Meyer, \& Georganopoulos. 2014, ApJL, 780, L27

[20] Meyer et al. 2015, ApJ, 805,154

[21] Mücke, \& Protheroe. 2001, Astroparticle Physics, 15, 121

[22] Mücke et al. 2003, Astroparticle Physics, 18, 593
[23] Nenkova et al. 2002, ApJL, 570, L9

[24] Peng et al. 2002, AJ, 124, 266

[25] Petropoulou, \& Dimitrakoudis. 2015, MNRAS, 452,1303

[26] Petropoulou et al. 2015, MNRAS, 448, 2412

[27] -. 2017, MNRAS, 464, 2213

[28] Plotkin et al. 2012, ApJL, 745, L27

[29] Roychowdhury et al. in prep.

[30] Sanchez et al. 2015, MNRAS, 454, 3229

[31] Stalevski et al. 2012, MNRAS, 420, 2756

[32] Urry, \& Mushotzky. 1982, ApJ, 253, 38

[33] Urry, \& Padovani. 1995, , 107, 803

[34] von Montigny et al. 1995, ApJ, 440, 525

[35] Zacharias, \& Wagner. 2016, Galaxies, 4, 63 